A patient with mononeuropathy multiplex, sensory neuronopathy, and diffuse intestinal pseudo-obstruction consisting of gastroparesis and

\section{Section Editors}

Robert C. Griggs, MD

Rochester, New York

Jerry R. Mendell, MD

Columbus, Ohio

Robert G. Miller, MD

San Francisco, California impaired small and large bowel motility was found to have a small cell carcinoma of the lung. The constellation of findings were indicative of paraneoplastic neuropathy, which was confirmed with the appropriate antibody studies demonstrating antineuronal nuclear antibodies. Identification of paraneoplastic neuropathy is important, since early treatment of the primary cancer, which is virtually always a small cell carcinoma of the lung, may halt the progression of the disorder. (c) 1994 John Wiley \& Sons, Inc.

Key words: paraneoplastic somatosensory - gastroparesis - pseudoobstruction - mononeuropathy multiplex • sensory neuropathy - antineuronal antibodies

MUSCLE \& NERVE $\quad$ 17:91-96 1994

\title{
PARANEOPLASTIC PSEUDO-OBSTRUCTION, MONONEUROPATHY MULTIPLEX, AND SENSORY NEURONOPATHY
}

BERTRAND C. LIANG, MD, JAMES W. ALBERS, MD, PhD, ANDERS A.F. SIMA, MD, PhD, and TIMOTHY T. NOSTRANT, MD

Paraneoplastic syndromes are the remote effects of cancer. These disorders indirectly affect the nervous system in an unpredictable fashion, usually causing substantial functional impairments. ${ }^{10}$ Other organ systems can be involved in these syndromes, although this is less common. We report a patient with paraneoplastic intestinal pseudoobstruction with presumed involvement of the myenteric plexus, and findings suggestive of a mononeuropathy multiplex and sensory neuropathy. The diffuse intestinal pseudo-obstruction included gastroparesis, marked small and large intestinal dilation, and poor propulsive activity particularly evident in the colon.

From the Departments of Neurology, Pathology (Neuropathology), and Internal Medicine (Gastroenterology), University of Michigan Medical Center, Ann Arbor, Michigan

Presented in part at the American Academy of Neurology, Conference on Neuromuscular Diseases, San Diego, California, May 4, 1992.

Acknowledgments: B.C.L. is the Susan Jacobson Feliow of the American Brain Tumor Association. We thank Vanda A. Lennon, MD, Director, Neuroimmunology Laboratory. Departments of Immunology and Neurology, Mayo Clinic, for performing the neuroimmunology studies reported for this patient

Address reprint requests to James $W$. Albers, MD, PhD, Department of Neurology, 1914 Taubman Center, University of Michigan Medical Center. Ann Arbor, Ml 48109-0316.

Accepted for publication May 1, 1993.

CCC 0148-639X/94/010091-06

(c) 1994 John Wiley \& Sons, Inc

\section{CASE REPORT}

The patient is a 66 -year-old male, who 7 months prior to admission developed nausea without dysphagia. His oral food intake decreased, and he began to lose weight. Six months prior to admission he underwent a left knee replacement for degenerative changes, and began noticing a tingling sensation in his fingertips, which progressed over weeks to involve his hands and forearms asymmetrically, the right side being more involved than the left. This was associated with distal and later proximal weakness. Shortly thereafter, he developed tingling in his distal lower extremities, which progressed over days, again the right side being more affected than the left. He was hospitalized in a psychiatric unit for presumed depression, where he remained for 6 weeks. He suffered a ruptured abdominal aortic aneurysm, and was transferred to another institution for repair of the aneurysm. Over the next 2 months he had progressive difficulty with eating because of abdominal pain, resulting in substantial weight loss. Two weeks prior to admission he developed acute urinary retention and was thought to have atypical chronic inflammatory demyelinating polyradiculoneuropathy (CIDP), based in part on reduced conduction velocities. An MRI of the spine showed cervical spondylosis but was otherwise normal; upper gastrointestinal endoscopy and colonoscopy were in- 
terpreted as unremarkable. Anti-GM $\mathrm{GM}_{1}$ antibodies were reportedly of "very high titer," and sedimentation rate was $58 \mathrm{~mm} / \mathrm{h}$. The patient was transferred to the University of Michigan Medical Center for further evaluation.

Past history revealed a remote and resolved pericarditis, a left nephrectomy $3 \mathrm{yr}$ prior to admission for renal cell carcinoma, treated hypertension, intermittent depression, and glaucoma. The patient gave a family history of coronary artery disease, cancer, and a sister with "swallowing difficulties." Social history was significant for a 90 pack/yr history of smoking. Medications on admission included ranitidine, metoclopramide, multivitamin (no pyridoxine), heparin subcutaneously, timolol, enalapril, morphine, and erythromycin.

On examination, the patient appeared chronically ill and uncomfortable. He was afebrile. Supine blood pressure was 128/78, upright blood pressure $112 / 66$, with pulse increasing by 21 beats per minute. On general examination there were high pitched bowel sounds and mild abdominal tenderness. Mental status was normal, as was examination of the cranial nerves. Strength examination (MRC scale; R/L) revealed: deltoids $4+14+$, biceps $4 / 4+$, triceps $4 / 4$, wrist extensors $3 / 4$, carpal interossei $3 / 4$, abductor pollicis brevis $3 / 4$, iliopsoas $4+/ 4+$, hamstrings $5 / 5$, leg flexion and extension
$5 / 5$, ankle dorsiflexion $4 / 5$, and ankle plantar flexion $5 / 5$. He showed decreased bulk and tone of the distal muscles of his hands and feet, right greater than left. There were no fasciculations. There was anesthesia to light touch and pin prick over the entire right upper extremity, decreased pin prick sensation over the right thorax and leg, and diminished vibration sense at the ankles. Position sense was decreased in the right distal fingers and toe joints. He was areflexic. Plantar stimulation produced a flexor response. Gait could not be examined because of weakness, abdominal discomfort, and orthostatic lightheadedness.

Hospital Course. The initial findings were thought to be consistent with a mononeuropathy multiplex and dysautonomia resulting in chronic pseudo-obstruction. Oral intake was discontinued and total parenteral nutrition was initiated. Upper gastrointestinal endoscopy revealed no masses and no spontaneous gastric activity. Nerve conduction studies (Table 1) revealed absent sensory nerve action potentials and asymmetric motor responses (right more abnormal than left) characterized by reduced compound muscle action potential amplitude without evidence of abnormal temporal dispersion or partial conduction block. There was no decrement to repetitive motor nerve stimulation at

\begin{tabular}{|c|c|c|c|c|c|c|c|c|c|c|}
\hline \multirow[b]{2}{*}{ Stimulate } & \multirow[b]{2}{*}{ Record } & \multicolumn{3}{|c|}{$A m p(m V$ or $\mu V)$} & \multicolumn{3}{|c|}{$\mathrm{CV}(\mathrm{m} / \mathrm{s})$} & \multicolumn{3}{|c|}{ Distal latency (ms) } \\
\hline & & Right & Left & Normal & Right & Left & Normal & Right & Left & Normal \\
\hline \multicolumn{11}{|l|}{ Median M } \\
\hline Wrist & Thenar & $0.3^{*}$ & 1.7 & $4-18$ & & & & 4.5 & 4.8 & $2.4-4.4$ \\
\hline Elbow & & 0.3 & 1.5 & & 53.4 & 49.0 & $49-70$ & & & \\
\hline Median $\mathrm{F}$ wave & & & & & & & & & 39.7 & $<31.0$ \\
\hline \multicolumn{11}{|l|}{ Ulnar $\mathrm{M}$} \\
\hline Wrist & Hypothenar & $0.4^{\star}$ & 4.6 & $6-16$ & & & & 4.9 & 3.7 & $1.8-3.5$ \\
\hline Elbow & & 0.3 & 4.0 & & 53.7 & 46.6 & $49-71$ & & & \\
\hline Ulnar $F$ wave & & & & & & & & NR & 37.2 & $<31.0$ \\
\hline \multicolumn{11}{|l|}{ Tibial M } \\
\hline Ankle & Abductor hallucis & $0.2^{*}$ & & $3-27$ & & & & 6.3 & & 3.3--6.1 \\
\hline \multicolumn{11}{|l|}{ Peroneal M } \\
\hline Ankle & & NR & & $2-12$ & & & & & & \\
\hline Peroneal & & & & & & & & NR & & \\
\hline Knee & Anterior tibialis & 4.5 & & $>3.0$ & & & & & & \\
\hline Median S & Index & NR & NR & $>15$ & & & & & & \\
\hline Ulnar S & Digit $V$ & NR & NR & $>10$ & & & & & & \\
\hline Radial S & Digit I & $N R$ & & $>10$ & & & & & & \\
\hline Sural S & Ankle & $N R$ & NR & $6-47$ & & & & & & \\
\hline SSR hand & Palm & NR & NR & $>1.0$ & & & & & & \\
\hline SSR foot & Foot & NR & & $>0.9$ & & & & & & \\
\hline
\end{tabular}

$M=$ motor,$S=$ sensory, $C V=$ conduction velocity, $S S R=$ sympathetic skin response, $N R=$ no response.

*No decrement to $3-\mathrm{Hz}$ repetitive stimulation; physiologic facilitation after exercise. 


\begin{tabular}{|c|c|c|c|c|}
\hline \multirow[b]{2}{*}{ Muscle } & \multicolumn{3}{|c|}{ Spontaneous activity } & \multirow[b]{2}{*}{ Motor unit action potentials } \\
\hline & Insertional activity & Fib & Fasc & \\
\hline L biceps brachii & Normal & 0 & 0 & Normal \#, A, D, \%P \\
\hline$L$ triceps & Normal & 0 & 0 & Normal \#, A, D, \%P \\
\hline L FDI (hand) & Sustained + waves & $1+$ & 0 & Slight $\downarrow \#, \uparrow A, D, \% P$ \\
\hline R FDI (hand) & Sustained + waves & $3+$ & 0 & Moderate $\downarrow \#, \uparrow A, D, \% P$ \\
\hline R abductor pollicis & Sustained + waves & $2+$ & 0 & Moderate $\downarrow \#, \uparrow A, D, \% P$ \\
\hline$L$ anterior tibialis & Unsustained + waves & 0 & 0 & Slight $\downarrow$ \#, $\uparrow A, D, \% P$ \\
\hline L medial gastrocnemius & Sustained + waves & $1+$ & 0 & Slight $\downarrow \#, \uparrow A, D, \% P$ \\
\hline$R$ anterior tibialis & Sustained + waves & $2+$ & 0 & Moderate $\downarrow \#, \uparrow A, D, \% P$ \\
\hline$A$ vastus medialis & Normal & 0 & 0 & Normal \\
\hline R medial gastrocnemius & Sustained + waves & $1+$ & 0 & Moderate $\downarrow$ \#, $\uparrow A$, normal $D, \% P$ \\
\hline$R$ internal hamstring & Normal & 0 & 0 & Normal \\
\hline R paraspinal low lumbar & Normal & 0 & 0 & Normal \\
\hline
\end{tabular}

$\#=$ Number of MUAPS, $A=$ amplitude, $D=$ duration, $\% P=$ percent polyphasic MUAPS, $\uparrow=$ increased, $\downarrow=$ decreased, fib $=$ fibrillation potentials, fasc = fasciculation potentials.

$3 \mathrm{~Hz}$ and only physiologic facilitation immediately after voluntary exercise. Needle electromyography (Table 2) demonstrated evidence of asymmetric, multifocal denervation and reinnervation. The combined electrodiagnostic studies were reported as consistent with a mononeuropathy multiplex superimposed upon a sensory neuronopathy and autonomic neuropathy.

Sural nerve biopsy revealed marked fiber loss, particularly of large myelinated fibers, and several fibers undergoing wallerian degeneration. Clusters of regenerating fibers were commonly encountered. There was no evidence of inflammation or vasculitis. Electron microscopic examination revealed, in addition, marked axonal atrophy of preserved myelinated fibers (Fig. 1). There also was marked loss of unmyelinated fibers with denervated Schwann cell profiles and atrophy of unmyelinated fibers (Fig. 2). Teased fiber preparation revealed $32.5 \%$ normal myelinated fibers, $41.8 \%$ showing regeneration, $13.9 \%$ wallerian degeneration, and $11.5 \%$ excessive myelin wrinkling indicative of axonal atrophy.

Biopsies from colonoscopy revealed several dysplastic polyps, as well as a rectal mass consisting of a tubulovillous adenoma with dysplastic features. Barium contrast only reached to the splenic flexure, demonstrating dilated loops of bowel. A CT scan of the chest (Fig. 3) revealed a questionable pathologic $1.5-\mathrm{cm}$ node in the right paratracheal region.

A trial of clear liquids was attempted, but all fluids were withdrawn by nasogastric suction $16 \mathrm{~h}$ later. The weakness and sensory dysfunction remained stable. Abdominal exploration demon- strated a splenic flexure adhesion, which was lysed, and another polyp which was removed. Postoperatively, there was no improvement of the patient's symptoms.

Additional laboratory studies demonstrated elevated antinuclear neuronal $\mathrm{I} / \mathrm{anti}-\mathrm{Hu}$ antibodies $(1: 61,490)$ and anticalcium channel antibodies. No antinuclear neuronal II or anti-Purkinje cell antibodies were present. The elevated titers supported the possibility of a small cell neoplasm. Because the search for a small cell neoplasm was otherwise negative, mediastinoscopy was performed, and a paratracheal lymph node biopsy revealed an in situ small cell carcinoma (Fig. 4). The patient subsequently received one course of systemic chemo-

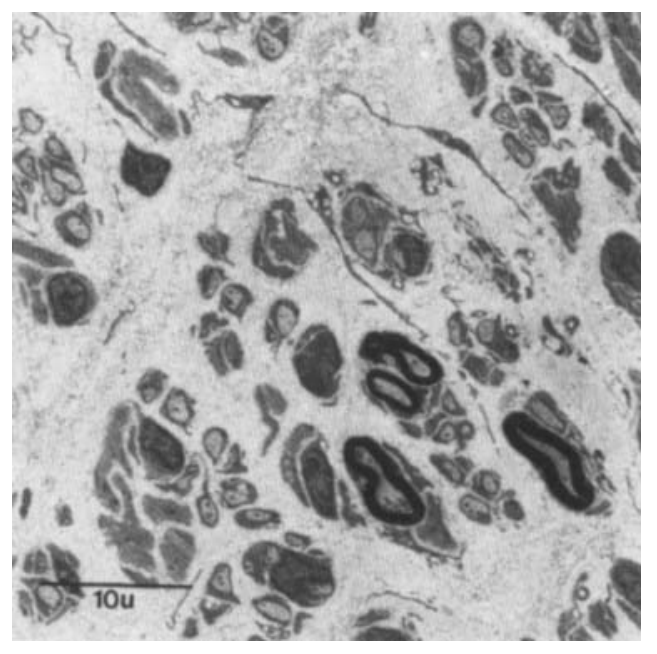

FIGURE 1. Electronmicrograph illustrating loss of large myelinated fibers. Preserved myelinated fibers show axonal atrophy. Magnification $3200 \times$. 


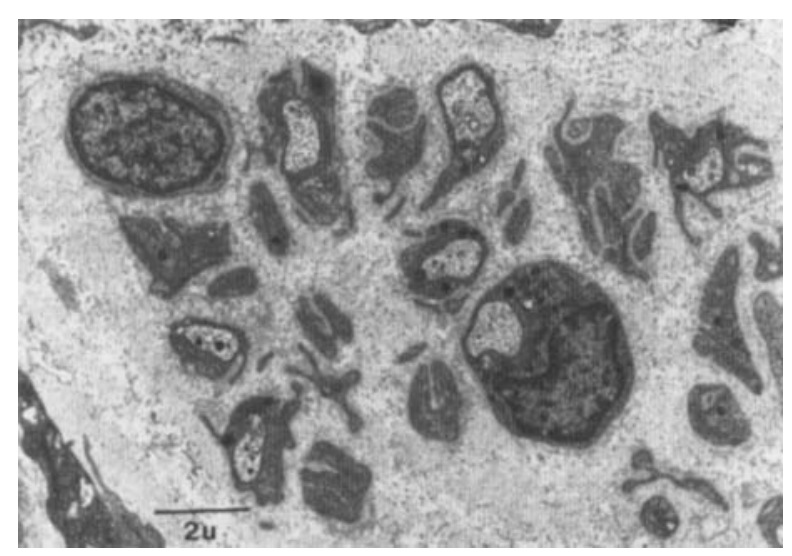

FIGURE 2. Electronmicrograph showing loss of unmyelinated fibers and denervated Schwann cell profiles. Note also atrophied unmyelinated fibers. Magnification $9900 \times$.

therapy (cyclophosphamide, doxorubicin, and etoposide), but refused any further therapy. His neurologic and gastrointestinal symptoms remained unchanged until his death 9 months later.

\section{DISCussion}

The neuromuscular findings in this patient presented a difficult challenge and initially were not thought to be suggestive of an underlying malignancy. The clinical presentation was characterized initially by progressive, multifocal, asymmetric impairment, and identified as a mononeuropathy multiplex. Identification of diffuse distal sensory loss was attributed to confluence of the mononeuropathy rather than a sensory neuronopathy. Sural nerve biopsy was performed looking for evidence of vasculitis or inflammation, and the absence of findings suggestive of vasculitis was unexpected, although the clinical course had not been one of

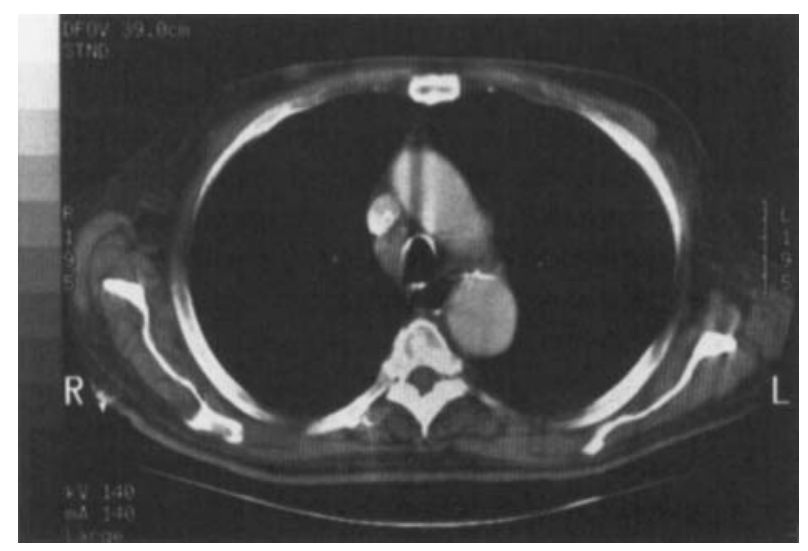

FIGURE 3. CT scan of chest revealing a $1.5-\mathrm{cm}$ lymph node in the paratracheal region. abrupt involvement of individual nerves. Sequential electrodiagnostic studies identified the sensory abnormalities as diffuse and severe, and also established evidence of dysautonomia as characterized by absent sympathetic skin responses. Idiopathic pseudo-obstruction refers to any intestinal dysfunction manifested by poor propulsive movement in the small intestine or colon simulating a mechanical obstruction but without any documented area of narrowing. In addition, there may be esophageal, gastric, and anal involvement. In this patient, small intestinal, colonic, and gastric involvement was documented. In retrospect, the diffuse pseudo-obstruction was the cardinal feature of this patient's presentation that suggested the possibility of a paraneoplastic syndrome, but initially this was thought to be unrelated to the neurologic disorder. It was weight loss, not gastroparesis and constipation, that initiated the initial negative search for neoplasm. Only when the neuropathy and pseudo-obstruction were associated with antinuclear neuronal antibody I was the search intensified and the small cell carcinoma identified.

Autonomic instability and paralysis of the digestive tract was first associated with cancer in 1975 by Ahmed and Carpenter. ${ }^{1}$ They described a patient with small cell carcinoma who had tumor infiltration into autonomic nerves, particularly in the myenteric plexus. In 1980 , Lhermitte et al. ${ }^{6}$ described a patient with small cell carcinoma of the lung and myenteric plexopathy, and suggested this being a "paraneoplastic syndrome." Schuffler et al., in $1983,{ }^{12}$ described a patient with chronic pseudo-obstruction and subsequent progressive autonomic dysfunction associated with small cell carcinoma of the lung, postulating an autoimmune etiology for the syndrome. Sodhi et al. ${ }^{13}$ reported 2 patients with intestinal pseudo-obstruction and small cell carcinoma of the lung. Autonomic function testing was consistent with partial autonomic dysfunction involving the pre- and postganglionic structures of the sympathetic and parasympathetic nervous systems. Chinn and Schuffler ${ }^{2}$ reported 7 additional patients with dysautonomia, myenteric plexopathy on biopsy, with pseudo-obstruction. Two patients manifested a constellation of neurologic disorders, including encephalopathy, ataxia, neurogenic bladder, and peripheral polyneuropathy, who developed their neurologic symptoms after the abdominal symptoms. In this report, the diagnosis of cancer was made 1-26 months after symptoms began. Five of 7 patients died within 9 


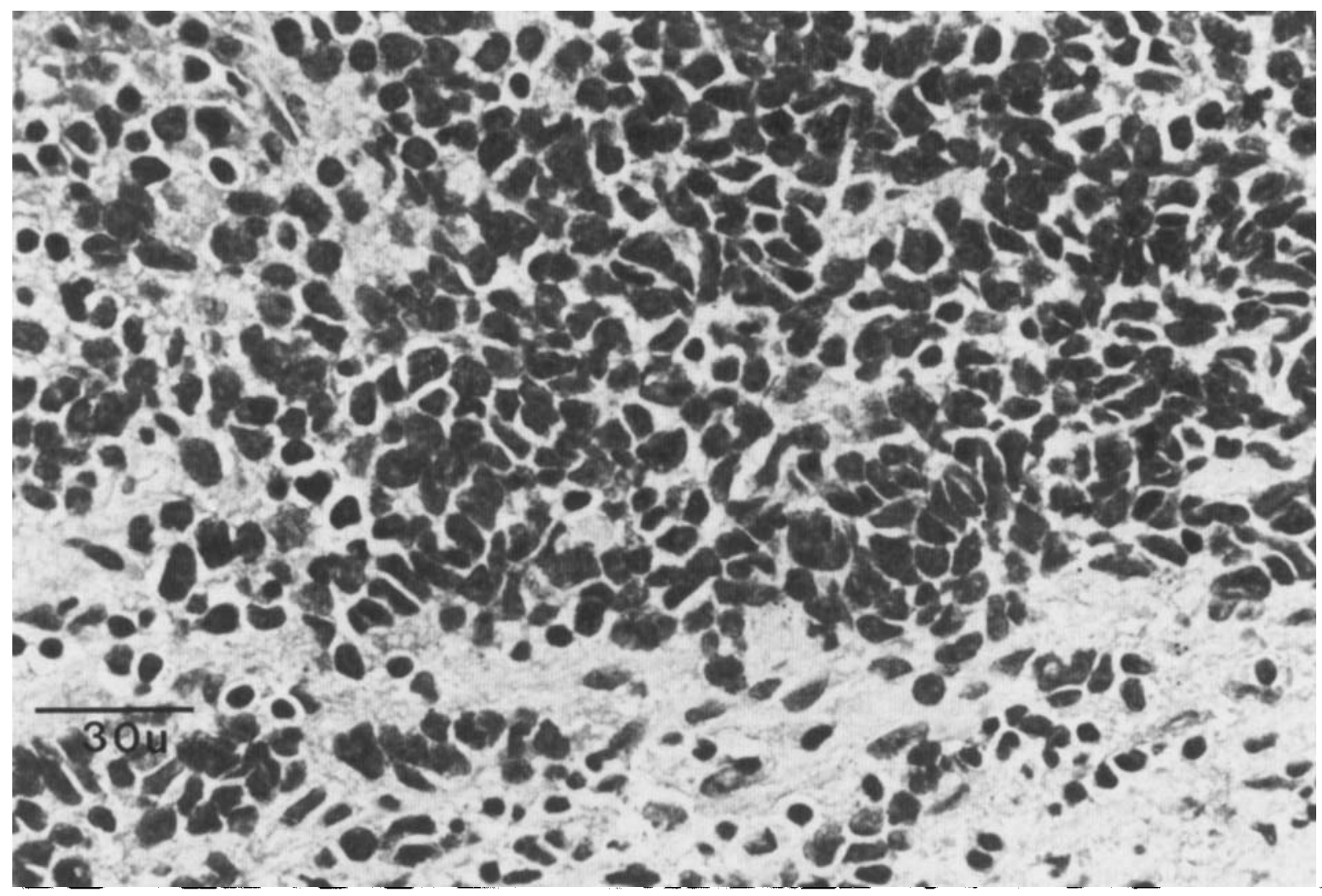

FIGURE 4. Micrograph showing small cell carcinoma of the biopsied mediastinal lymph node. Magnification $620 \times$.

months of the diagnosis of chronic pseudoobstruction; the remaining 2 patients were alive after 41 and 57 months. In 1991, Lennon and colleagues ${ }^{5}$ associated myenteric plexopathy and autonomic neuropathy with a specific antibody, antinuclear neuronal antibody I, which reacted to antigens in the nuclei of neuronal cells ${ }^{5}$ as well as with antigens from cerebellum, dorsal root ganglion, brain, and spinal cord. These myenteric antibodies also reacted with antigens found in small cell carcinoma of the lung, and were identical to the anti-Hu antibody associated with sensory neuropathy. ${ }^{4}$ Hence, paraneoplastic pseudo-obstruction became recognized as a novel paraneoplastic syndrome with an antibody marker also associated with paraneoplastic sensory neuropathy and encephalitis. The appearance of multifocal mononeuropathy as a paraneoplastic syndrome is controversial, although peripheral vasculitis with a resultant mononeuritis multiplex has been associated with small cell lung carcinoma ${ }^{3}$ and renal cell carcinoma. ${ }^{14}$ The pathogenesis is unknown and there is no antibody marker as yet to identify this syndrome. Nevertheless, we were unable to dem- onstrate evidence of an underlying vasculitis, and the presence of a mononeuropathy multiplex in this patient does not establish the association with the underlying neoplasm.

The clinical presentation of paraneoplastic pseudo-obstruction usually begins with gastrointestinal symptoms of progressive anorexia, early satiety, vomiting, and constipation; patients usually complain of gastrointestinal discomfort, and often reveal a history of an abdominal surgical procedure and substantial weight loss. ${ }^{2}$ After several weeks to months, paresthesias of the distal extremities begin, progressing to a severe peripheral neuropathy with weakness, postural instability, and urologic symptoms. Reported cases demonstrate a predominance of either peripheral or autonomic symptoms, although most patients had evidence of involvement of both systems.

The differential diagnosis of paraneoplastic pseudo-obstruction relates to that of any disorder of intestinal motility. The first entity to exclude is mechanical ("true") obstruction. Subsequently, causes for pseudo-obstruction or dysmotility should be assessed. Primary chronic pseudo- 
obstruction is uncommon, and has been related to visceral myopathy and inclusion body myenteric plexopathy. ${ }^{9}$ There are familial forms of pseudoobstruction inherited in autosomal-dominant and autosomal-recessive patterns. ${ }^{9}$ More frequently, the etiology of pseudo-obstruction is secondary, and includes connective tissue diseases (e.g., myositis, scleroderma, lupus, amyloidosis), endocrine disorders (e.g., hypothyroidism, diabetes, hypoparathyroidism), medications (due to anticholinergic effects), or other neurologic diseases which affect the autonomic nervous systems (e.g., ShyDrager syndrome or paraneoplastic pseudoobstruction). ${ }^{9}$

The identification of paraneoplastic pseudoobstruction is important in order to initiate the search for a primary cancer and appropriate treatment. Treatment of the cancer may halt the progression of the dysautonomia, ${ }^{7,8,13}$ although reversal of symptoms is uncommon. Symptomatic therapy for pseudo-obstruction is limited, since pharmacologic approaches to enhance bowel motility are relatively ineffective. Total parenteral nutrition and supportive therapy are the primary treatments. ${ }^{11}$ Although most patients do not regain intestinal motility, some do, and periodic retrials of oral feedings may be indicated to identify such patients.

The prognosis for patients with paraneoplastic pseudo-obstruction is poor. Most patients die within a year after diagnosis without regaining either neurologic or gastrointestinal function; however, several reports include patients who survived and had some return of gastrointestinal, but not neurologic, function. ${ }^{2,13}$ Although no specific prognostic signs have been identified, early identification of the syndrome most likely portends to the best prognosis, since therapy for the primary cancer may halt progression.

In summary, we have reported a patient with diffuse pseudo-obstruction, a mononeuropathy multiplex, and a sensory neuropathy who was found to have a small cell carcinoma of the lung. The association of antinuclear neuronal antibody I with the sensory neuropathy and gastroparesis was important in promoting the search for an under- lying malignancy. Paraneoplastic pseudo-obstruction should be considered in any patient with a subacute course of gastroparesis associated with peripheral nervous system findings. Early identification of the syndrome is important, since treatment of the primary cancer, which is almost always a small cell carcinoma of the lung, may halt the progression of the syndrome.

\section{REFERENCES}

1. Ahmed MN, Carpenter S: Autonomic neuropathy and carcinoma of the lung. Can Med Assoc J 1975;1 13;410:412.

2. Chinn JS, Schuffler MD: Paraneoplastic visceral neuropathy as a cause of severe gastrointestinal motor dysfunction. Gastroenterology 1988;95:1279-1286.

3. Johnson PC, Rolak AL, Hamilton RH, Laguna JF: Paraneoplastic vasculitis of nerve: a remote effect of cancer. Ann Neurol 1979;5:437-444.

4. Kiers L, Altermatt HJ, Lennon VA: Paraneoplastic antineuronal nuclear IgG autoantibodies (type I) localize antigen in small cell lung carcinoma. Mayo Clin Proc 1991;66: $1209-1216$.

5. Lennon VA, Sas DF, Busk MF, Scheithaver B, Malagelada JR, Camillieri M, Milner LJ: Enteric neuronal autoantibodies in pseudo-obstruction with small cell lung carcinoma. Gastroenterology 1991;100:737-742.

6. Lhermitte F, Gray F, Lyon-Caen O, Pertuiset BF, Bernard $P$ : Paralysis of digestive tract with lesions of myenteric plexus: a new paraneoplastic syndrome. Rev Neurol (Paris) 1980;136:825-836.

7. Malagelada JR, Camillieri M, Stanghellini V: Manometric Diagnosis of Gastrointestinal Motility Disorders. New York, Thieme, 1986 .

8. Park DM, Johnson RH, Cream GP, Robinson JF: Orthostatic hypotension in bronchial carcinoma. Br Med J 1972; 3:510-511.

9. Phillips S: Disorders of gastrointestinal motility, in Wyngaarden JB, Smith LH (ed): Cecil Textbook of Medicine. Philadelphia, Saunders, 1985, pp 702-712.

10. Posner JB: Paraneoplastic syndromes involving the nervous system, in Aminoff MJ (ed): Neurology and General Medicine. New York, Churchill Livingstone, 1989, pp 341-364.

11. Schuffler M: Diagnosis and treatment of chronic idiopathic intestinal pseudo-obstruction syndromes. Sarvey Dig Dis 1984;2:104-114.

12. Schuffler MD, Baird HW, Fleming CR, Bell CE, Bouldin TW, Malagelada JR, McGill DB, Le Bauer SM, Abrams M, Love J: Intestinal pseudo-obstruction as the presenting manifestation of small cell carcinoma of the lung. Ann Int Med 1983;98:129-134.

13. Sodhi N, Camillieri M, Camoriano JK, Low PA, Fealey RD, Perry MC: Autonomic function and motility in intestinal pseudo-obstruction caused by paraneoplastic syndrome. Dig Dis Sci 1989;34:1937-1942.

14. Torvic A, Berrtzen AE: Necrotizing vasculitis without visceral involvement. Acta Med Scand 1968;184:69-78. 